\title{
Theoretical Insights Into the Impact of Coherent and Incoherent Crosstalk on Optical DPSK Signals
}

\author{
João J. O. Pires, Member, IEEE, and Luís G. C. Cancela, OSA
}

\begin{abstract}
This paper provides new theoretical insights into the properties of direct detection differential phase-shift keying (DPSK) signals impaired by coherent and incoherent crosstalk. Coherent crosstalk is due to multiple replicas originated while a data signal is routed through an optical network, whereas the source of incoherent crosstalk resides on the interference from other DPSK signals. A special emphasize has been put on modeling the multipath coherent crosstalk, with analytical expressions being derived and presented for both the moment generating function of the decision variable and the average error probability. A rigorous analysis, capable of dealing with arbitrary filtering, is also presented, which is used afterwards to assess the accuracy of the analytical formulas. A detailed comparison with incoherent crosstalk is also performed. Using also an exact treatment for this type of crosstalk it is shown that for low OSNR penalties the coherent crosstalk leads, in some circumstances, to slightly worse results than the incoherent one, but this situation is reversed when the total crosstalk level and the number of interferers increase.
\end{abstract}

Index Terms-Coherent crosstalk, differential phase-shift keying (DPSK), error analysis, incoherent crosstalk, optical networks.

\section{INTRODUCTION}

I $\mathrm{N}$ the past few years, differential phase-shift keying (DPSK) has attracted considerable attention in the field of long haul optical transmission due to its advantages over on-off keying $(\mathrm{OOK})$ in such aspects as a higher receiver sensitivity, an improved robustness to transmission impairments and a better tolerance to power fluctuations [1]. In addition, there is also considerable interest about how this scheme performs in the context of optical transparent networks (i.e., networks with no optical-electrical-optical conversion in the intermediate nodes). In these networks, the optical channels are switched in the optical domain and, consequently, the physical limitations of the optical devices employed in the network nodes can originate light leakages that interfere with the desired signal originating crosstalk. It is well known that this phenomenon can be particularly damaging when the interference and the signal have the same nominal wavelength, leading to the so-called in-band crosstalk [2].

Manuscript received August 20, 2009; revised March 18, 2010; accepted June 04, 2010. Date of publication August 09, 2010; date of current version September 08, 2010.

J. J. O Pires is with Instituto de Telecomunicações and Department of Electrical and Computer Engineering, Instituto Superior Técnico, 1049-001 Lisboa, Portugal (e-mail: jpires@1x.it.pt).

L. G. C. Cancela is with Instituto de Telecomunicacções and Department of Science and Information Technology, Instituto Superior de Ciências do Trabalho e da Empresa, 1649-026 Lisboa, Portugal (e-mail: luis.cancela@ iscte.pt).

Digital Object Identifier 10.1109/JLT.2010.2064285
In-band crosstalk can be classified as either coherent or incoherent [3]. The first form of crosstalk, also denoted as multipath coherent crosstalk, arises when the interfering terms are weak delayed replicas of the main signal and the differential delays between the signal and its replicas are much smaller than the laser coherence time. On the other hand, the incoherent counterpart arises when the interferers and the main signal come from different laser sources, or when the differential delays are far larger than the laser coherence time. Due to the coherent nature of the interaction between the signal and interfering fields, the amplitude of the received power in the presence of coherent crosstalk will fluctuate in time scales that are much longer than the bit period. This phenomenon is similar to the signal fading encountered in mobile communications environments and, in the same way, it can be a source of burst errors [4]. On the contrary, the time scales of the power fluctuations due to incoherent crosstalk are much shorter than the bit period and, as a consequence, this impairment is inherently a source of random errors. The singularities of multipath crosstalk place new challenges in the area of performance estimation, since the traditional deterministic power penalties associated with incoherent crosstalk can no longer be used.

The architectures of the optical nodes, the type of components used, and the way how these components are interconnected determine, to a great extent, the forms of crosstalk generated inside these nodes and the interplay between them. Taking, for example, as a reference the optical cross-connect architecture presented in [5], the space switches are responsible for creating incoherent crosstalk, whereas the (de)multiplexer stages create both coherent and incoherent terms. Another important network element is the optical add-drop multiplexer (OADM). Focusing on an OADM based on arrayed-waveguide gratings in a loop-back configuration [6] one observes that the channels that pass through the nodes are contaminated by coherent crosstalk due to the loop-back paths, whereas the incoherent crosstalk affects the added and dropped channels. In general, the reconfigurable OADMs are more susceptible to the effects of coherent crosstalk than the static configurations, whereas the levels of incoherent crosstalk are more dependable on the OADM structure in the static configurations than in the reconfigurable ones [7]. Finally, it is also worth mentioning that in the optical space switching matrices besides the typical incoherent crosstalk due to the presence of multiple input signals with the same wavelength, there is also coherent crosstalk as a result of the multiple propagation paths inside the switch due to the finite isolation of the switching elements [8].

The intrinsic analog nature of optical transparent networks even exacerbate the crosstalk problem, in the measure that the 
crosstalk generated inside each node accumulates as the signal corresponding to a given channel traverses multiple network nodes [9]. In networks with a large number of nodes and a high wavelength density this impairment can become the main source of performance degradation and a major scaling limitation factor.

The incoherent in-band crosstalk has been extensively analyzed in the literature, especially in the context of OOK systems (see [2] and references therein), and has also aroused some interest on scenarios based on DPSK [10]-[12], due to the fact of DSPK signals being more resilient to the impact of this impairment than OOK signals [10]. On the contrary, only a few studies have dealt with multipath coherent crosstalk [13], [14], and only recently its impact on direct detection DPSK systems has been assessed [15]. In [13] it is emphasized that coherent crosstalk causes fading of the received optical power and shown by experimental and simulation means that the $Q$-factor fluctuates in a stochastic way. The fact of the coherent crosstalk being the cause of random fluctuations on the received optical power is also evidenced by other authors [5], [14]. The later reference also provides analytical and numerical probability density functions (PDFs) for the received powers and demonstrates that in the presence of coherent crosstalk the power penalties used to characterize the system performance are no longer deterministic, as happens in scenarios based on the incoherent crosstalk. Reference [15] uses some of the findings reported in [13] and [14], namely the fact of the received optical signal in presence of multipath interference could be described using Rician statistics, as the basis for developing a new treatment for evaluating the impact of coherent crosstalk on DPSK signals and derives analytical formulas for both the moment generating function (MGF) of the decision variable and error probability, by using the well-known wideband optical filtering assumption [16] to model the optical receiver.

In this paper, we provided a rigorous formulation to assess the simultaneous implications of coherent crosstalk and amplified spontaneous emission (ASE) noise on DPSK signals, by extending the results presented in [15] in order to deal with arbitrary filtering. The treatment requires the decomposition of the signal, coherent crosstalk, and ASE noise in terms of a series of orthogonal functions and relies on using eigenfunction expansion techniques. The derivation of the closed-form expression for the average error probability, given in [15], is also detailed. Furthermore, to make the paper self-contained we also briefly describe a closely related method to rigorously compute the error performance in the presence of incoherent crosstalk, which will be used afterwards to compare the impact of the two types of crosstalk on DPSK signals.

This paper is structured as follows. Section II gives some background related to the decomposition of the decision variable in terms of a series of orthogonal functions. Section III describes an exact analysis for evaluating the impact of coherent crosstalk and provides also analytical expressions. Section IV briefly describes a rigorous method for performance estimation in the presence of incoherent crosstalk using symbol conditioning. Numerical results are given in Section V and, finally, some conclusions are presented in Section VI.

\section{RECEIVER CONFIGURATION AND EIGENFUNCTION EXPANSION}

In our analyses we have assumed that the received DPSK signals are processed using a direct detection optical receiver, which includes an optical pre-amplifier, an optical filter, a Mach-Zehnder delay interferometer with a differential delay equal to the bit period $T$, a balanced photodetector, and an electrical filter. The pre-amplifier is considered to have flat gain $G$ and be a source of ASE noise. This noise is described as a zero mean white stationary Gaussian noise with single-sided power spectral density per polarization given by $N_{o}=h \nu_{s}(G-1) F / 2$, where $h \nu_{s}$ is the photon energy at the signal frequency and $F$ is the noise figure. The optical filter is assumed to have an arbitrary equivalent low-pass impulse response $h_{o}(t)$ and an optical bandwidth $B_{o}$, whereas the electrical filter is described by the impulse response $h_{e}(t)$ and by the electrical bandwidth $B_{e}$. In this work, the impact of the filters on the system performance is described in a compact form by using the parameter

$$
\Lambda(t, \tau)=\int_{-\infty}^{+\infty} h_{e}(\varsigma) h_{o}(t-\varsigma) h_{o}(\tau-\varsigma) d \varsigma
$$

The filters responses can affect the signal, the crosstalk, and the ASE noise properties. As a result, it is important to consider in the analyses realistic filter shapes such as that of a Gaussian shape. Actually, a Gaussian optical filter is a good model to describe real optical filter shapes [17] and it is well known that the frequency responses of the typical Bessel electrical filters and Gaussian filters are very close, especially for higher filter orders. The combination of a Gaussian shaped optical filter with an electrical filter with the same shape, denoted here as Gaussian receiver, will be used widely in this paper. Another approach also used to simplify the performance analyses is the wideband optical filtering approximation. This approximation involves the use of an ideal rectangular optical filter with a large bandwidth-time product $\left(B_{O} T\right)$ and an electrical integrate-and-dump filter with $h_{e}(t)=1 / T$ for $t \in[0, T]$ and zero elsewhere.

The properties and the performance of DPSK signals can be studied using either PDFs or error probabilities. Both these metrics can be derived from the MGF of the decision variable, so the knowledge of this function is a central piece in the statistical description of the receiver output samples. Usually, to evaluate the MGF of the decision variable it is required that this variable be written as a summation of independent random variables, which implies the use of eigenfunction expansion techniques to decompose the signal, the interference, and the ASE noise in terms of a series of orthogonal functions. This can be done by choosing for the orthogonal functions a set of eigenfunctions $\left\{\varphi_{k}(t)\right\}_{k=0}^{\infty}$ of the integral equation

$$
\int_{-\infty}^{+\infty} \Lambda(t, \tau) \varphi_{k}(\tau) d \tau=\lambda_{k} \varphi_{k}(t)
$$

where $\left\{\lambda_{k}\right\}_{k=0}^{\infty}$ are the corresponding eigenvalues. An additional advantage coming from using a Gaussian receiver configuration is the possibility of obtaining closed-form expressions 
for both the eigenfunctions and eigenvalues, without requiring the numerical evaluation of (2) [16].

\section{Multipath Coherent Crosstalk Model}

\section{A. Modeling and Exact Expressions}

In order to characterize the multipath coherent crosstalk, let us consider an optical channel that carries a DPSK signal at the optical frequency $\nu_{s}$. This channel is routed through a number of network nodes which are responsible for adding multipath coherent crosstalk. This crosstalk comes from multiple propagation paths inside the nodes due to unwanted light leakage from non-ideal components, such as optical filters and optical switches. At the channel output, the DPSK signal is processed using a direct detection receiver. In the presence of $N_{c}$ interfering fields coming from the multipath propagation, the electrical field of the DPSK signal can be described during the interval $[0, T]$ by

$$
\begin{aligned}
\vec{E}_{s}(t)= & \sqrt{2 P_{o}} u(t) \exp \left[j 2 \pi \nu_{s} t+j \theta_{s}(t)+j \phi_{s}(t)\right] \vec{r}_{s} \\
& \times\left\{1+\sum_{i=1}^{N_{c}} \sqrt{\varepsilon_{i}} \frac{u\left(t-\tau_{i}\right)}{u(t)} \exp \left(-j 2 \pi \nu_{s} \tau_{i}\right)\right. \\
& \times \exp \left[j\left(\theta_{s}\left(t-\tau_{i}\right)-\theta_{s}(t)\right)\right. \\
& \left.\left.+j\left(\phi_{s}\left(t-\tau_{i}\right)-\phi_{s}(t)\right)\right] \frac{\vec{r}_{i}}{\vec{r}_{s}}\right\}
\end{aligned}
$$

where $P_{o} \propto\left|E_{o}\right|^{2}$ denotes the average optical power corresponding to the main field with amplitude $E_{o}, u(t)$ a rectangular pulse of unitary amplitude within the interval $[0, T]$ and zero elsewhere, $\phi_{s}(t)$ the phase noise, $\theta_{s}(t)$ the signal phase conveying the information, $\varepsilon_{i}^{1 / 2}$ and $\tau_{i}$ are the attenuation and the differential delay relative to the signal of the $i$ th interfering field, respectively, $\vec{r}_{s}$ and $\vec{r}_{i}$ are the polarization unit vectors of the signal and the $i$ th interfering field, respectively. The signal phase is given by $\theta_{s}(t)=\theta_{s}(t-T)+\pi\left(1-a_{s}\right) / 2$, where $\theta_{s}(t-T)$ is the phase in the previous time interval, and $a_{s}=1$ for symbol "one" and $a_{s}=-1$ for the symbol "zero". The parameter $\varepsilon_{i}$ is the ratio between the optical power of the $i$ th interfering term and the signal optical power and is also denoted here as the crosstalk level. To simplify (3) a number of assumptions can be made: 1) the differential delay $\tau_{i}$ is much smaller than the bit period implying that $\theta_{s}(t) \approx \theta_{s}\left(t-\tau_{i}\right)$ and $\left.u(t) \approx u\left(t-\tau_{i}\right) ; 2\right)$ the differential delay $\tau_{i}$ is also much smaller than the laser coherence time and, as a consequence, $\left.\phi_{s}(t) \approx \phi_{s}\left(t-\tau_{i}\right) ; 3\right)$ all the interfering fields suffer the same attenuation, i.e., $\left.\varepsilon_{i}^{1 / 2}=\varepsilon^{1 / 2} ; 4\right)$ the signal and all the interfering fields are co-polarized, i.e., $\vec{r}_{i}=\vec{r}_{s}$. It is convenient to note that the hypothesis of co-polarization between the fields leads to a worst case performance. Similarly, assumptions 1) and 3) also maximize the impact of crosstalk. However, if the delay $\tau_{i}$ is larger than the bit period, then $u(t)$ and $u\left(t-\tau_{i}\right)$ become completely uncorrelated, which reduces the impact of coherent crosstalk. This property was used, for example, in [18] to devise a scheme appropriate to reduce the effects of coherent crosstalk in OADMs. Under the above assumptions, the complex envelope of the electrical field of the signal plus ASE noise at the interferometer input is given by

$$
\tilde{E}(t)=\left\{\sqrt{2 G P_{s}} u(t) \exp \left[j \theta_{s}(t)+j \phi_{s}(t)\right]\right\} \vec{r}_{s} * h_{o}(t)+\tilde{E}_{n}(t)
$$

where $*$ denotes convolution, $\tilde{E}_{n}(t)$ describes the complex envelope of the filtered ASE noise originated from the optical pre-amplifier, and $P_{s} \propto\left|E_{s}\right|^{2}$ with

$$
E_{s}=E_{o}\left[1+\sqrt{\varepsilon} \sum_{i=1}^{N_{c}} \exp \left(-j \varphi_{i}\right)\right]
$$

where $\varphi_{i}=2 \pi \nu_{s} \tau_{i}$ is the phase offset of the $i$ th interfering field relative to the signal. The phase $\varphi_{i}$ varies in a random manner following a uniform distribution between $[0,2 \pi]$, as a consequence of drifts in the laser central frequency, or changes in the differential delay coming from thermal or mechanical fluctuations.

This situation is similar to the one encountered in mobile communication systems, when a strong signal suffers interference from many random weaker components that leads to the well-known Rician fading [19]. Therefore, the optical channel can also be modeled as a Rician fading channel and the optical power $P_{s}$ described as a random process. Assuming that the number of interfering fields is high, as well as that all the fields have the same amplitude and their phases are mutually independent, then it can be shown from (5) that (see Appendix A) the PDF of $P_{s}$ is described by

$$
p\left(P_{s}\right)=\frac{K}{P_{o}} \exp (-K) \exp \left(\frac{-K P_{s}}{P_{o}}\right) I_{0}\left(2 K \sqrt{\frac{P_{s}}{P_{o}}}\right)
$$

where $K$ is the Rician parameter given by $K=1 /\left(N_{c} \varepsilon\right), I_{0}(x)$ is the modified Bessel function of first kind and order zero. As seen, the Rician parameter is exactly the inverse of the total crosstalk level $\varepsilon_{T}=N_{c} \varepsilon$, so in a channel without crosstalk $K=\infty$. Furthermore, we assume that the gain $G$ is sufficiently high, so that the ASE noise dominates over shot noise and thermal noise in the receiver, allowing us to neglect these noises in the present analysis. It is worthy of note that Rician statistics can be used to model a number of situations encountered in realistic scenarios, such as the routing of an optical channel through a cascade of OADMs in the presence of interference due to adjacent channels [13], or the switching of an optical channel through an optical space switching fabric, whenever the transmission path is subject to multipath propagation [8].

The statistic of the decision variable $V$ at the electrical filter output, defined at the decision time $t_{d}$, can be described in terms of the MGF $M_{V}(s)$, defined as the expected value of $e^{s V}$. The MGF of $V$, conditioned on the optical power $P_{s}$, can be expressed in terms of the MGF of the random variable $V^{+}$, corresponding to the constructive port of the interferometer, and the MGF of the random variable $V^{-}$ 
corresponding to the destructive port, in the following way $M_{V}\left(s \mid P_{s}\right)=M_{V^{+}}\left(s \mid P_{s}\right) M_{V^{-}}\left(-s \mid P_{s}\right)$ with [16]

$$
\begin{aligned}
& M_{V^{ \pm}}\left(s \mid P_{s}\right) \\
& \quad=\frac{1}{\prod_{k=0}^{\infty}\left(1-s \lambda_{k} N_{o} / 2\right)} \exp \left(\sum_{k=0}^{\infty} \frac{s \lambda_{k} \xi_{k} T G P_{s} \alpha_{s}^{ \pm}}{1-s \lambda_{k} N_{o} / 2}\right)
\end{aligned}
$$

where $\xi_{k}=u_{k}^{2} / T$ with $u_{k}=\int_{-\infty}^{+\infty} u\left(t_{d}-\tau\right) \varphi_{k}(\tau) d \tau$, and $\alpha_{s}^{ \pm}=\left(1 \pm a_{s}\right)^{2} / 4$, providing that the phase noise can be neglected. In the above equations $\varphi_{k}(t)$ is the $k$ th eigenfunction and $\lambda_{k}$ the corresponding eigenvalue of (2). The unconditional MGF of $V$ is now given by

$$
M_{V}(s)=M_{V^{+}}(s) M_{V^{-}}(-s)
$$

where $M_{V \pm}(s)$ is evaluated by integrating (7) over the PDF of $P_{s}$, giving (9), shown at the bottom of the page.

\section{B. Simplifications and Closed-Form Expressions}

An expedite way to simplify the expression of $M_{V}(s)$ consists in considering the wideband optical filtering approximation. This simplification is obtained by making the following approximations [16]: 1) $\lambda_{k}=1 / T$, for $k<B_{o} T$ and zero elsewhere; 2) $\sum_{k=0}^{B_{o} T-1} \xi_{k}=1$. In these circumstances, (9) can be simplified giving for the symbol "one"

$$
\begin{aligned}
M_{V}(s)= & \frac{1}{\left(1-2 s \sigma^{2}\right)^{M}\left(1+2 s \sigma^{2}\right)^{M}} \frac{1}{\left[\frac{-s G P_{o}}{K\left(1-2 s \sigma^{2}\right)}+1\right]} \\
& \times \exp \left[\frac{\frac{s G P_{o}}{1-2 s \sigma^{2}}}{\frac{-s G P_{o}}{K\left(1-2 s \sigma^{2}\right)}+1}\right]
\end{aligned}
$$

where $M=B_{o} T$ and $\sigma^{2}=N_{o} /(4 T)$. The average error probability can be derived from (10) with the help of the classical inverse Laplace transform ([19], (13)). However, in this work we follow a different approach, since we first obtain an expression for the conditional error probability and then average the obtained equation over $P_{s}$, as explained in more detail in Appendix B. As a result, one arrives to the following closed-form expression for the average error probability:

$$
\begin{aligned}
P_{e}= & \frac{1}{2^{n+1}} \frac{1}{\left(\gamma_{c}+1\right)} \exp \left(-\frac{\gamma}{\gamma_{c}+1}\right) \sum_{k=0}^{n} a_{k}(n) \\
& \times\left(\frac{\gamma_{c}}{\gamma_{c}+1}\right)^{k} L_{k}\left(-\frac{K}{\gamma_{c}+1}\right)
\end{aligned}
$$

where

$$
a_{k}(n)=\sum_{i=k}^{n} \frac{1}{2^{i}}\left(\begin{array}{c}
n+i \\
i-k
\end{array}\right)
$$

In (11) $n=M-1, \gamma$ is the optical signal-to-noise ratio (OSNR) evaluated using a noise bandwidth equal to the bit rate, given by $\gamma=G P_{o} T / N_{o}, \gamma_{c}=\gamma / K$, and $L_{k}(x)$ is the Laguerre polynomial of degree $k$. A number of special cases are of particular interest. Firstly, for an ideal matched optical filter, $M=1$, and (11) reduces to

$$
P_{e}=\frac{1}{2} \frac{1}{\left(\gamma_{c}+1\right)} \exp \left(-\frac{\gamma}{\gamma_{c}+1}\right) .
$$

Furthermore, when the multipath crosstalk can be neglected $(K=\infty)$, one sees that (13) leads to the well-known expression for DPSK, this is

$$
P_{e}=\frac{1}{2} \exp (-\gamma)
$$

Finally, for a channel without coherent crosstalk and for $M=2$, (11) leads to

$$
P_{e}=\frac{1}{2} \exp (-\gamma)\left[1+\frac{\gamma}{4}\right]
$$

which is similar to the expression reported in ([11], (5)) for an optical matched filter reception in the presence of the two ASE noise polarizations.

\section{INCOHERENT CROSSTALK MODEL}

To model the incoherent crosstalk let us suppose that the received DPSK signal, at the output of a given channel, is impaired by in-band crosstalk due to $N$ DPSK interferers, originated from $N$ different sources with the same bit rate and nominal frequency as the desired signal $\left(\nu_{s}\right)$. Therefore, the complex envelope of the total electrical field at the interferometer input can be given by

$$
\tilde{E}(t)=\tilde{E}_{r}(t)+\sum_{i=1}^{N} \tilde{E}_{x, i}(t)+\tilde{E}_{n}(t)
$$

where the first term corresponds to the desired signal, the second term to the incoherent in-band crosstalk, and the third term, as in the coherent crosstalk case, to the ASE noise originated from the optical pre-amplifier. $\tilde{E}_{r}(t)$ is obtained from (3) neglecting the multipath interference, giving

$$
\tilde{E}_{r}(t)=\left\{\sqrt{2 G P_{o}} u(t) \exp \left[j \theta_{s}(t)+j \phi_{s}(t)\right] \vec{r}_{s}\right\} * h_{o}(t)
$$

whereas

$$
\tilde{E}_{x, i}(t)=\left\{\sqrt{2 G P_{x, i}} u(t) \exp \left[j \theta_{x, i}(t)+j \phi_{x, i}(t)\right] \vec{r}_{i}\right\} * h_{o}(t)
$$

$$
M_{V^{ \pm}}(s)=\frac{1}{\prod_{k=0}^{\infty}\left(1-s \lambda_{k} N_{o} / 2\right)} \frac{1}{\left(\frac{-\alpha_{s}^{ \pm} G T P_{o}}{K} \sum_{k=0}^{\infty} \frac{s \lambda_{k} \xi_{k}}{1-s \lambda_{k} N_{o} / 2}+1\right)} \exp \left[\frac{\alpha_{s}^{ \pm} G T P_{o} \sum_{k=0}^{\infty} \frac{s \lambda_{k} \xi_{k}}{1-s \lambda_{k} N_{o} / 2}}{\frac{-\alpha_{s}^{ \pm} G T P_{o}}{K} \sum_{k=0}^{\infty} \frac{s \lambda_{k} \xi_{k}}{1-s \lambda_{k} N_{o} / 2}+1}\right]
$$


where $P_{x, i}$ is the average crosstalk power at the amplifier input, $\theta_{x, i}(t)=\theta_{x, i}(t-T)+\pi\left(1-a_{x, i}\right) / 2$ is the phase conveying the information of the $i$ th interfering signal, with $a_{x, i}=1$ for the symbol "one" and $a_{x, i}=-1$ for the symbol "zero", and $\phi_{x, i}$ is a random phase. The crosstalk level of the $i$ th interferer $\varepsilon_{i}$ is defined in the same way as in the previous section, permitting to write $\varepsilon_{i}=P_{x, i} / P_{o}$, whereas the total crosstalk level is given here by $\varepsilon_{T}=\sum_{i=1}^{N} \varepsilon_{i}$.

In this situation, the evaluation of the MGF of the random variable $V$ is also a two steps process. In the first place, it is evaluated the MGF conditioned on the phase difference $\Delta \theta_{i}(t)=$ $\theta_{x, i}(t-T)-\theta_{s}(t-T)+\phi_{x, i}(t)-\phi_{s}(t)$. Secondly, the obtained MGF is averaged over the distribution of $\Delta \theta_{i}$, which is assumed to be uniformly distributed over $[0,2 \pi]$. As a consequence the $M_{V}(s)$ is obtained using (8) with [20]

$$
M_{V^{+}}(s)=\frac{1}{\prod_{k=0}^{\infty}\left[1-s \lambda_{k} N_{o} / 2\right]} M_{y_{1}}\left[\sum_{k=0}^{\infty} \frac{s \lambda_{k} T \xi_{k}}{\left(1-s \lambda_{k} N_{o} / 2\right)}\right]
$$

and

$$
M_{V^{-}}(s)=\frac{1}{\prod_{k=0}^{\infty}\left[1-s \lambda_{k} N_{o} 2\right]} M_{y_{2}}\left[\sum_{k=0}^{\infty} \frac{s \lambda_{k} T \xi_{k}}{\left(1-s \lambda_{k} N_{o} / 2\right)}\right] .
$$

In these equations the $M_{y_{1}}(s)$ is given by

$$
\begin{aligned}
M_{y_{1}}(s)= & \exp \left(s G P_{o} \alpha_{s}^{+}\right) \\
& \times \prod_{i=1}^{N} \exp \left(s G P_{o} \varepsilon_{i} \alpha_{x, i}^{+}\right) I_{0}\left(s 2 G P_{o} \sqrt{\varepsilon_{i} \alpha_{s}^{+} \alpha_{x, i}^{+}}\right)
\end{aligned}
$$

where $\alpha_{x, i}^{+}=\left(1+a_{x, i}\right)^{2} / 4$, whereas $M_{y_{2}}(s)$ is given by (21) with $\alpha_{s}^{+}$replaced by $\alpha_{s}^{-}$and $\alpha_{x, i}^{+}$replaced by $\alpha_{x, i}^{-}=(1-$ $\left.a_{x, i}\right)^{2} / 4$. It is also expected that the fact of a certain interferer, for instance interferer $i$, being at the state "one" $\left(a_{x, i}=1\right)$, or at the state "zero" $\left(a_{x, i}=-1\right)$ will condition the statistics of $V$. As a consequence, an additional refinement is required in the modeling process by taking into account all possible combinations of the symbol states of the interfering terms. In this case, we have

$$
M_{V}(s)=\frac{1}{2^{N+1}} \sum_{l=0}^{N}\left(\begin{array}{c}
N \\
l
\end{array}\right) M_{V}(s \mid l)
$$

where $M_{V}(s \mid l)$ is the conditional MGF given that the number of interferers in state "one" is $l$. Bearing in mind that for $l$ interferers in the state "one" there are $N-l$ in the state "zero", and using (8), the conditional MGF turns out to be $M_{V}(s \mid l)=$ $M_{V^{+}}(s \mid l) M_{V^{-}}(-s \mid(N-l))$.

\section{RESUlTS AND DISCUSSION}

The numerical results given here are obtained using the rigorous methods described in the previous sections to deal with the coherent and incoherent in-band crosstalk, considering a Gaussian receiver with the associated electrical filter having a 3 -dB bandwidth equal to $B_{e}=0.7 / T$. All these numerical results were computed using a finite number of eigenvalues, which depends on the bandwidth-time product. For instance, 12 eigenvalues were used for $B_{o} T=1$, and 24 for $B_{o} T=5$. Furthermore, the pre-amplifier is characterized by $G=30 \mathrm{~dB}$ and $F=5 \mathrm{~dB}$, whereas the operating bit rate is equal to $10 \mathrm{~Gb} / \mathrm{s}$. The simplified approach derived for the coherent crosstalk in Section III.B is also employed in our numerical calculations and its accuracy is assessed using the exact results obtained for the Gaussian receiver as a reference.

The PDF of decision variable is used frequently in this section, since this is a key function to provide a proper understanding of the statistical properties of DPSK signals. This function is computed by taking the inverse Laplace transform of the MGF of $V$. For a perfectly balanced DPSK receiver the PDFs corresponding to the symbols "one" and "zero" are identical and symmetric around the optimum decision threshold, which corresponds to $V=0$ [21]. For this reason, we will only consider in this analysis the PDFs of the decision variable for the symbol "one", which is normalized with respect to $P_{o}$.

Fig. 1 depicts these PDFs considering both the coherent and incoherent crosstalk with the latter one computed by assuming the presence of 16 interferers. This figure also includes curves for the coherent crosstalk obtained using the simplified approach. Focusing on the inner tails of the curves and on values of the decision variable lower than zero, since these are the fundamental elements to determine the error probability, one observes that, for the case of $B_{o} T=1$ (Fig. 1(a)), there is little difference between all the curves. This behavior gives the indication that the simplified formulation describes quite well not only the coherent crosstalk tails, but also the incoherent ones. The panorama is different in Fig. 1(b), where the tails corresponding to the simplified formulation deviates from the tails computed using the rigorous approaches, suggesting that for $B_{o} T=5$ the approximation lacks accuracy in the region under study. Similar observations have been drawn in [16], where the discrepancies were explained using the eigenfunctions and eigenvalues of the integral equation (2), but in an environment without crosstalk.

Fig. 2 plots the exact PDFs of the decision variable considering a total crosstalk level of $-17 \mathrm{~dB}$, assumed to be equally distributed among a variable number of interferers for the incoherent crosstalk case. A common feature to all the curves is their asymmetry, which gives a clear indication that the statistics of the received signal in the presence of the impairments under consideration have a non-Gaussian behavior. The asymmetry is more pronounced for the coherent curves, since their outer tails deviates markedly from the ones corresponding to the incoherent case. Interestingly, however, the inner tails of the former curves closely mimic those of the incoherent curves, especially when the number of interferers is sufficiently high. In addition, Fig. 2 also shows that the shape of the PDFs enlarges when the bandwidth-time product $B_{o} T$ increases from 1 to 5 . This enlargement is due to an increase in ASE noise power resulting from the augmentation of the optical filter bandwidth.

The dependence of the OSNR penalty, which is measured against back-to-back transmission, for an error probability of $10^{-9}$ on the total crosstalk level $\varepsilon_{T}$ for both the coherent and incoherent crosstalk is plotted in Fig. 3. The average error probability is computed by employing the rigorous expressions 


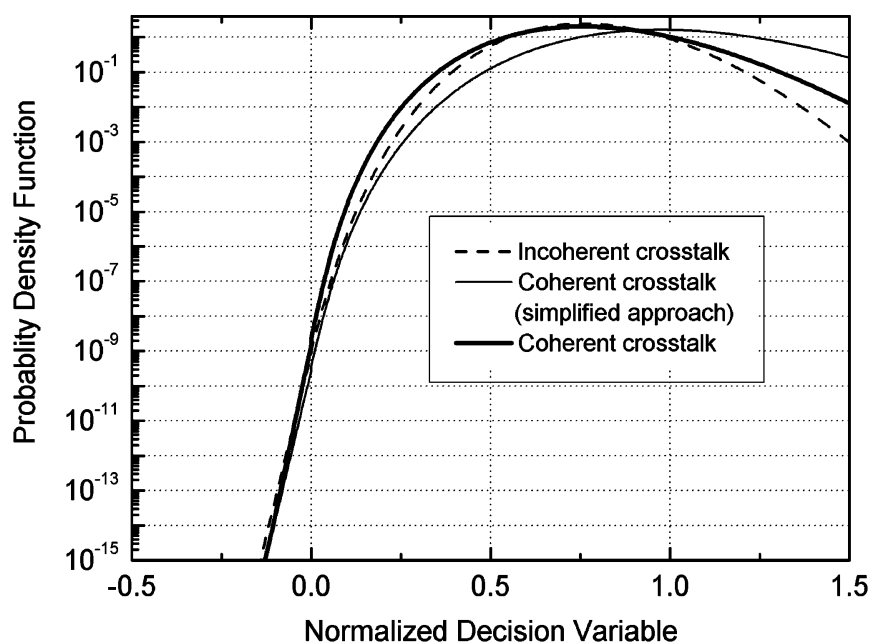

(a)

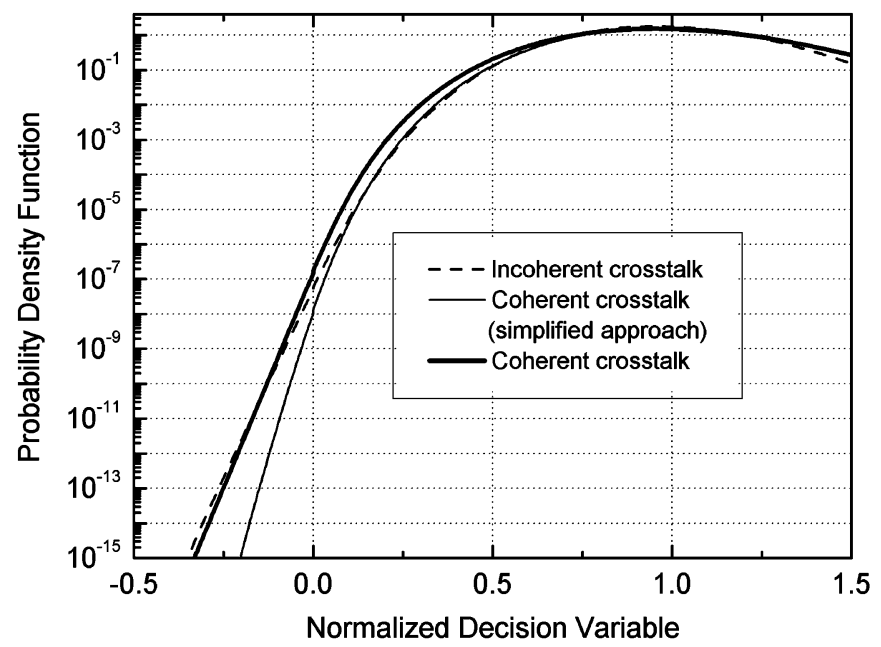

(b)

Fig. 1. PDF of the normalized decision variable for the symbol "one" in the presence of coherent and incoherent crosstalk considering a Gaussian receiver, $N=16, \varepsilon_{T}=-17 \mathrm{~dB}$, and OSNR $=17 \mathrm{~dB}$. Results are also shown for the coherent case using the simplified approach. (a) $B_{o} T=1$; (b) $B_{o} T=5$.

of the MGFs, derived in the previous sections, and applying standard saddle point integration techniques by using, for example, ([19], (13)). For $B_{o} T=5$ the results show that for a 1-dB OSNR penalty the crosstalk requirements for coherent crosstalk are more stringent than for the incoherent crosstalk, since the first impairment requires a crosstalk level of -19.8 $\mathrm{dB}$ whereas for the second one a value of $-19 \mathrm{~dB}$ is needed for the case of 16 interferers. In contrast, for $B_{o} T=1$ a close coincidence can be seen between the curves corresponding to the two types of crosstalk, provided that the number of interferers is equal or higher than 16 and the power penalties smaller than $2 \mathrm{~dB}$. In Fig. 3, we also investigate the accuracy of modeling the penalty curves corresponding to the coherent crosstalk by using the analytical expression for the error probability derived from the wideband filtering approximation, this is (11). For $B_{o} T=1$, this expression reduces to (13), which, despite its simplicity, yields results that are in excellent agreement with that obtained by relying on the rigorous formulation for the Gaussian receiver. It is worth noting that (13) can also be used

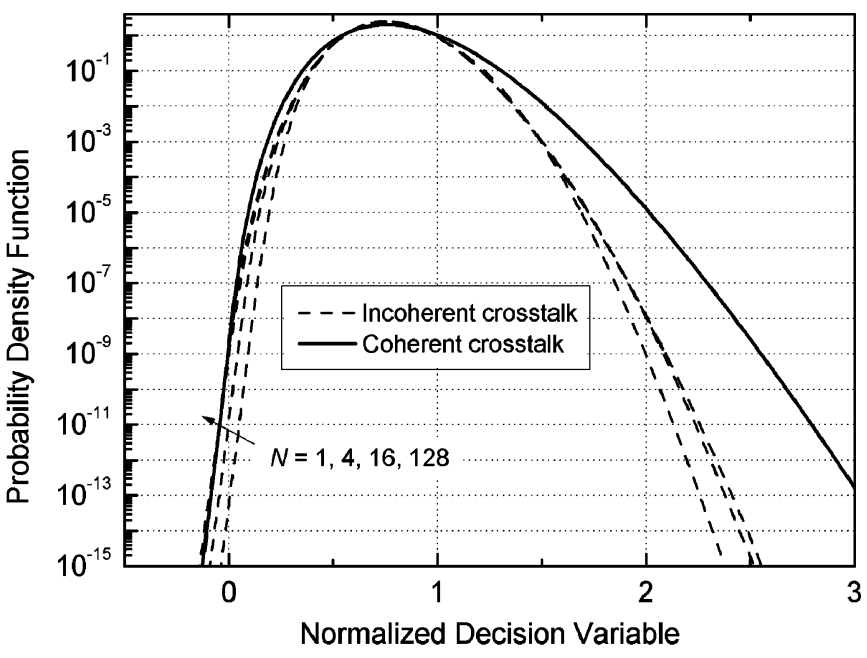

(a)

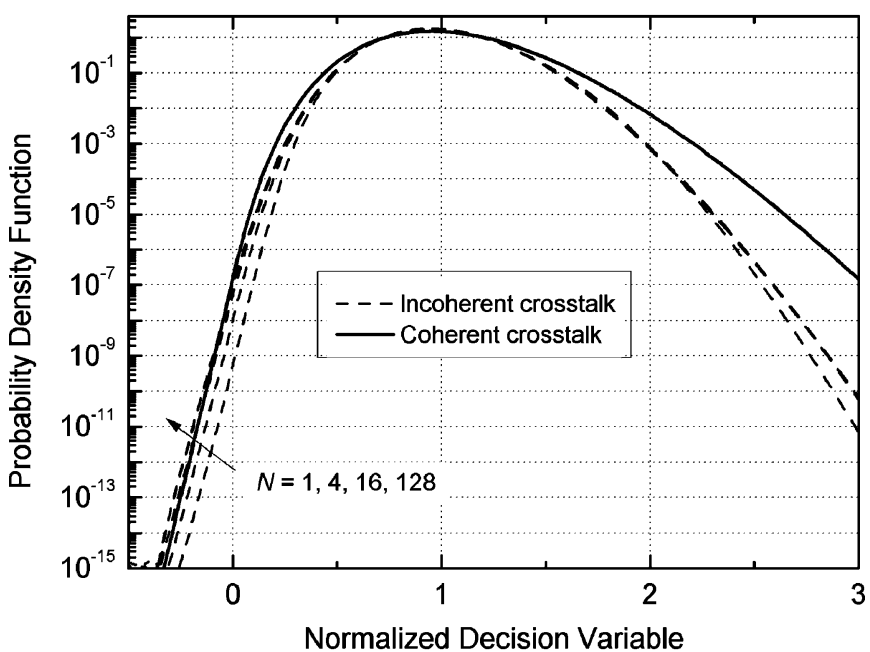

(b)

Fig. 2. PDF of the normalized decision variable for the symbol "one" in the presence of coherent and incoherent crosstalk considering a Gaussian receiver, $\varepsilon_{T}=-17 \mathrm{~dB}$ and OSNR $=17 \mathrm{~dB}$. In the incoherent case the total crosstalk level is equally distributed between a variable number of interferers. (a) $B_{o} T=$ $1 ;$ (b) $B_{0} T=5$.

to compute OSNR penalties in incoherent crosstalk scenarios in an expedite way, provided that the number of interferers is high and the range of penalties does not go beyond $2 \mathrm{~dB}$. For what concerns $B_{o} T=5$, the closed-form expression still predicts for the coherent crosstalk results close to the ones achieve by using the more complex and computational intensive method based on the rigorous MGF. But, in this case, the formula lacks accuracy in dealing with the incoherent crosstalk.

Finally, in Fig. 4 we present additional curves for the OSNR penalty, but now the target error probability is $10^{-3}$. As expected, the OSNR penalty is considerably lower at this new value of $P_{e}$, with the coherent crosstalk requiring only, $\varepsilon_{T}=$ $-13.8 \mathrm{~dB}$ and $\varepsilon_{T}=-14 \mathrm{~dB}$ for a $1-\mathrm{dB}$ penalty for $B_{o} T=1$ and $B_{O} T=5$, respectively. The degradation of the error probability is due to an increase in the ASE noise power, which in turn is responsible for mitigating the crosstalk effects and reducing the OSNR penalties due to crosstalk. In this new scenario, the discrepancies between the curves obtained using the exact and 


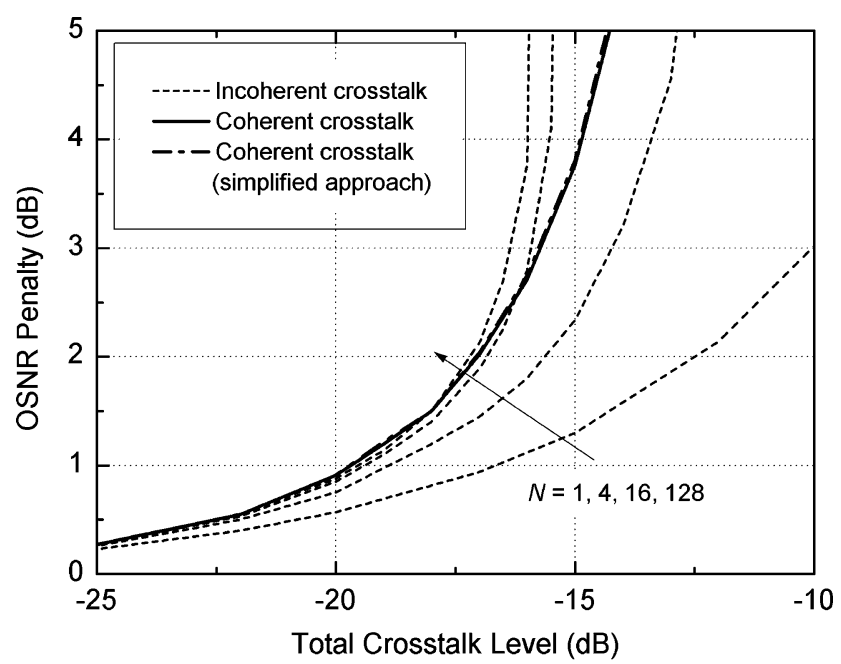

(a)

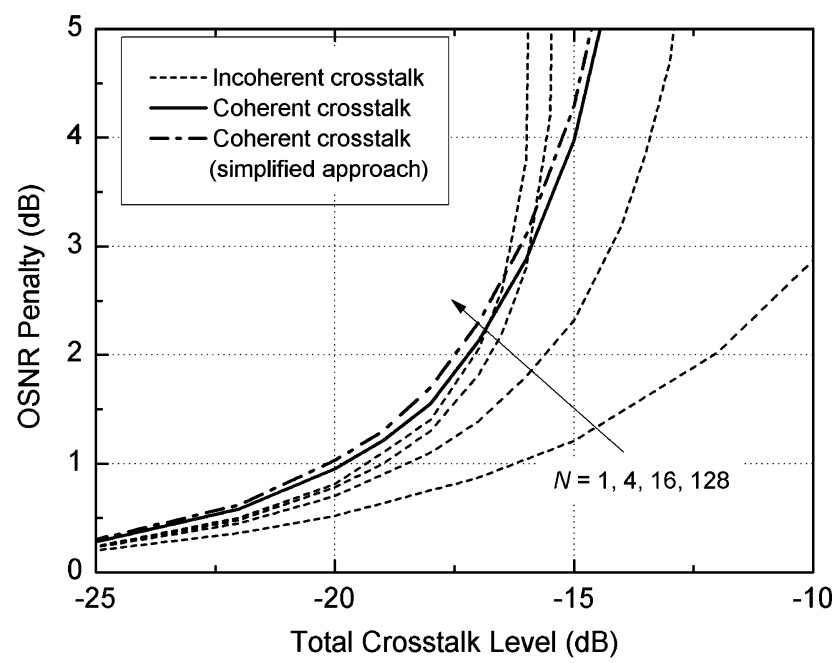

(b)

Fig. 3. OSNR penalty versus the total crosstalk level for both coherent and incoherent crosstalk at $P_{e}=10^{-9}$ considering a Gaussian receiver. Results are also shown for the coherent case using the simplified approach, i.e., with the error probability computed using (11). (a) $B_{o} T=1$; (b) $B_{o} T=5$.

the simplified approach to model the coherent crosstalk are still small, and here this crosstalk is less detrimental than the incoherent one, especially if we focus on OSNR penalties larger than $1-\mathrm{dB}$ and on a higher number of interferers.

\section{CONCLUSION}

In this paper, the effect of coherent and incoherent crosstalk on optical DPSK signals has been analyzed. A special emphasize has been put on modeling the coherent crosstalk resulting from multipath propagation inside the network nodes, by resorting to Rician statistics to describe the received optical power. A rigorous procedure appropriate for performance studies and able to deal with the case of arbitrary filtering, was presented. Furthermore, analytical studies, based on the wideband optical filtering approximation, were also carried out and a closed-form expression for the error probability was derived. Such approaches were then compared and shown that for what

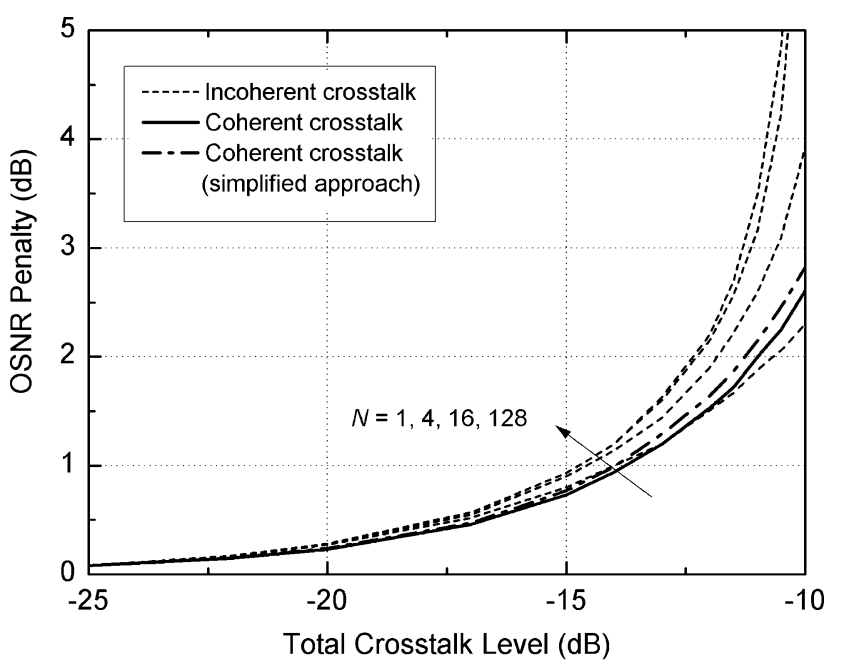

(a)

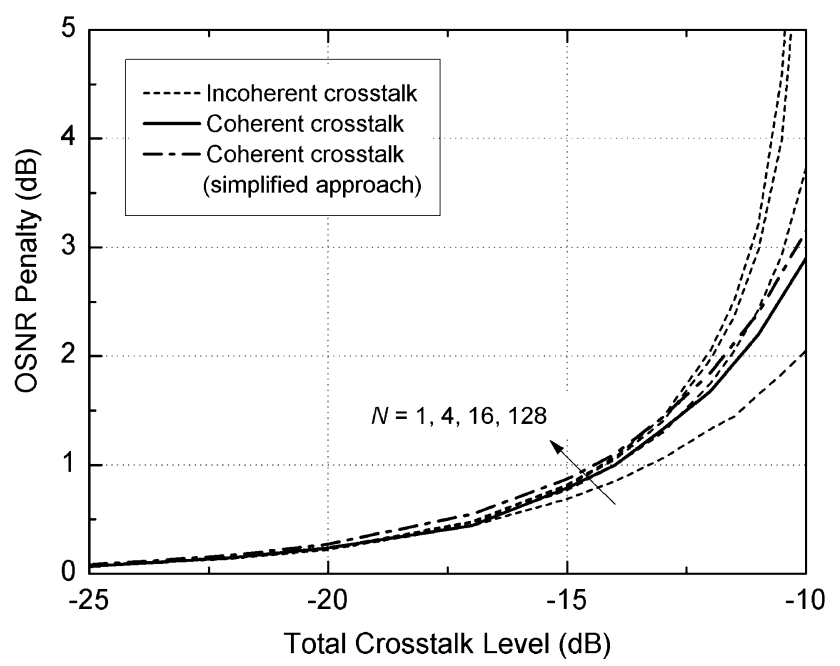

(b)

Fig. 4. Same as Fig. 3, but with the OSNR penalty evaluated at $P_{e}=10^{-3}$.

concerns the OSNR penalties the analytical formulation leads to quite accurate results. A modeling of the incoherent crosstalk using a rigorous analysis was also briefly described in order to get a clear picture about the relative impact of the two types of crosstalk. It is shown that for low OSNR penalties the level of stringency of any type of crosstalk depends on the number of interferers and on the bandwidth-time product and even on the value of the target error probability, but when the total crosstalk level and the number and interferers increase the incoherent crosstalk is always the most detrimental phenomenon.

\section{APPENDIX A}

Derivation of the Probability Density FunCtion of THE OPTICAL POWER $P_{S}$

From (5) it can be shown that

$$
\left|E_{s}\right|=\sqrt{\left(E_{o}+E_{x}\right)^{2}+E_{y}^{2}}
$$


where

$$
\begin{aligned}
& E_{x}=\sqrt{\varepsilon} E_{o} \sum_{i=1}^{N_{c}} \cos \left(\varphi_{i}\right) \\
& E_{y}=\sqrt{\varepsilon} E_{o} \sum_{i=1}^{N_{c}} \sin \left(\varphi_{i}\right) .
\end{aligned}
$$

By assuming that $N_{c}$ is large enough so that the central limit theorem can apply, then $E_{x}$ and $E_{y}$ can be described as two independent Gaussian distributed random variables with zero means. As the phase offsets $\varphi_{i}$ are uniformly distributed on $[0,2 \pi]$, then it can be shown that the variances of $E_{x}$ and $E_{y}$ are given by [14]

$$
\sigma_{x}^{2}=\sigma_{y}^{2}=\sigma_{s}^{2}=\frac{N_{c} \varepsilon E_{o}^{2}}{2} .
$$

In these circumstances $\left|E_{s}\right|$ is a Rician distributed random variable with its PDF given by the following equation [14], [22]:

$$
p\left(\left|E_{s}\right|\right)=\frac{\left|E_{s}\right|}{\sigma_{s}^{2}} \exp \left[\frac{-\left(\left|E_{s}\right|^{2}+E_{o}^{2}\right)}{2 \sigma_{s}^{2}}\right] I_{0}\left(\frac{\left|E_{s}\right| E_{o}}{\sigma_{s}^{2}}\right)
$$

where $I_{0}(x)$ is the modified Bessel function of the first kind and order zero. Note that (A5) is identical to the one used to describe the envelope statistics of the Rician fading [23]. Since $P_{s} \propto\left|E_{s}\right|^{2}$, then the PDF of $P_{s}$ can be obtained from (A5) by a simple change in variable, leading to (6).

\section{APPENDIX B}

\section{Derivation of the ERror Probability IN THE PRESENCE OF}

\section{Multipath COHERENT CROSSTALK}

The error probability for the DPSK conditioned on the optical power $P_{s}$ can be obtained from (7) by applying the simplification associated with the wideband assumption and using afterwards ([19], (13)), giving

$$
P_{e / P_{s}}=\frac{1}{2^{n+1}} \exp \left(-\frac{G P_{s} T}{N_{o}}\right) \sum_{k=0}^{n} \frac{a_{k}(n)}{k !}\left(\frac{G P_{s} T}{N_{o}}\right)^{k}
$$

where $n=M-1$ and $a_{k}(n)$ is defined in the text by (12). Note that (B1) can also be obtained by adapting to DPSK the expression presented by Humblet et al.. ([24], (9)) for the frequency-shift keying modulation. To evaluate the average error probability one must average the expression (B1) over the statistics of $P_{s}$ by making

$$
P_{e}=\int_{-\infty}^{+\infty} P_{e / P_{s}} p\left(P_{s}\right) d P_{s}
$$

Substituting (6) and (B1) into (B2) results in

$$
\begin{aligned}
P_{e} & =\frac{1}{2^{n+1}} \sum_{k=0}^{n} \frac{1}{k !} a_{k}(n) \int_{0}^{\infty} \exp \left(-\frac{G P_{s} T}{N_{o}}\right)\left(\frac{G P_{s} T}{N_{o}}\right)^{k} \\
& \times \frac{1}{2 \sigma_{c}^{2}} \exp [-K] \exp \left[\frac{-P_{s}}{2 \sigma_{c}^{2}}\right] I_{0}\left(2 \sqrt{K} \sqrt{\frac{P_{s}}{2 \sigma_{c}^{2}}}\right) d P_{s}
\end{aligned}
$$

where $2 \sigma_{c}^{2}=P_{o} / K$. Similarly to [25], we make in (B3) the following substitution $K^{\prime}=(K) /\left(2 C \sigma_{c}^{2}\right)$ and change the integration variable as $P_{s}=(z) /\left(2 C \sigma_{c}^{2}\right)$ with $C=\left((G T) /\left(N_{o}\right)+\right.$ $\left.(1) /\left(2 \sigma_{c}^{2}\right)\right)$. This leads to

$$
\begin{aligned}
& P_{e}=\frac{1}{2^{n+1}} \sum_{k=0}^{n} \frac{1}{k !} a_{k}(n)\left(\frac{G T}{C 2 \sigma_{c}^{2} N_{o}}\right)^{k} \exp [\left.-K+K^{\prime}\right] \\
& \times \frac{1}{C 2 \sigma_{c}^{2}}\left\langle z^{k}\right\rangle
\end{aligned}
$$

where $\left\langle z^{k}\right\rangle$ can be seen as the moment of order $k$ of the random variable $z$, described by

$$
\begin{aligned}
\left\langle z^{k}\right\rangle=\frac{1}{2 \sigma_{c}^{2}} \exp \left[-K^{\prime}\right] \int_{0}^{+\infty} z^{k} \exp & {\left[\frac{-z}{2 \sigma_{c}^{2}}\right] } \\
\times & I_{0}\left(2 \sqrt{\frac{K^{\prime} z}{2 \sigma_{c}^{2}}}\right) d z .
\end{aligned}
$$

Using ([26], (6.643.2)) we obtain after some algebra

$$
\left\langle z^{k}\right\rangle=k !\left(2 \sigma_{c}^{2}\right)^{k} \exp \left[-K^{\prime}\right]_{1} F_{1}\left(k+1 ; 1 ; K^{\prime}\right)
$$

where ${ }_{1} F_{1}(a ; b ; x)$ is the confluent hypergeometric function. This expression can be written in a more compact form by noting that the Laguerre polynomial of degree $k L_{k}(x)$ is related to the hypergeometric function through $L_{k}(x)={ }_{1} F_{1}(-k ; 1 ; x)$, and applying the relationship $e^{x}{ }_{1} F_{1}(a ; b ;-x)={ }_{1} F_{1}(b-a ; b ; x)$, derived from ([26], (9.212.1)). Then, we have

$$
\left\langle z^{k}\right\rangle=k !\left(2 \sigma_{c}^{2}\right)^{k} L_{k}\left(-K^{\prime}\right)
$$

As a consequence (B4) reduces to

$$
\begin{aligned}
P_{e}=\frac{1}{2^{n+1}} \sum_{k=0}^{n} a_{k}(n) & \left(\frac{G T}{C N_{o}}\right)^{k} \\
& \times \exp \left[-K+K^{\prime}\right] \frac{1}{C 2 \sigma_{c}^{2}} L_{k}\left(-K^{\prime}\right)
\end{aligned}
$$

Inserting $C, K^{\prime}$ and $\sigma_{c}^{2}$ into (B8) one arrives to (11).

\section{REFERENCES}

[1] A. H. Gnauck and P. J. Winzer, "Optical phase-shift-keyed transmission," J. Lightw. Technol., vol. 23, no. 1, pp. 115-130, Jan. 2005.

[2] J. C. Attard, J. E. Mitchell, and C. J. Rasmussen, "Performance analysis of interferometric noise due to unequally powered interferers in optical networks," J. Lightw. Technol., vol. 23, no. 4, pp. 1692-1703, Apr. 2005. 
[3] P. J. Legg, M. Tur, and I. Andonovic, "Solution paths to limit interferometric noise induced performance degradation in ASK/direct detection lightwave networks," J. Lightw. Technol., vol. 14, no. 9, pp. 1943-1954, Sep. 1996.

[4] C.-X. Wang and M. Pätzold, "A novel generative model for burst error characterization in Rayleigh fading channels," Proc. IEEE PIMRC'O3, pp. 960-964, 2003.

[5] Y. Shen, K. Lu, and W. Gu, "Coherent and incoherent crosstalk in WDM optical networks," J. Lightw. Technol., vol. 17, no. 5, pp. 759-764, May 1999.

[6] J. J. O. Pires, M. O’Mahony, N. Parnis, and E. Jones, "Scaling limitations in full-mesh WDM ring networks using arrayed-waveguide grating OADMs," Electron. Lett., vol. 35, no. 1, pp. 73-75, Jan. 1999.

[7] S. D. Dods and R. S. Tucker, "A comparison of the homodyne crosstalk characteristics of optical add-drop multiplexers," J. Lightw. Technol., vol. 19, no. 12, pp. 1829-1838, Dec. 2001.

[8] J. J. O. Pires and L. G. C. Cancela, "Coherent multipath crosstalk in Benes and dilated Benes optical switches," in Proc. LEOS'97, Nov. 1997, pp. 544-545.

[9] E. L. Goldstein and L. Eskildsen, "Scaling limitations in transparent optical networks due to low-level crosstalk," IEEE Photon. Technol. Lett., vol. 7, no. 1, pp. 93-94, Jan. 1995.

[10] X. Liu, Y.-H. Kao, M. Movassaghi, and R. C. Giles, "Tolerance to in-band coherent crosstalk of differential phase-shift-keyed signal with balanced detection and FEC," IEEE Photon. Technol. Lett., vol. 16, no. 4, pp. 1209-1211, Apr. 2004.

[11] K.-P. Ho, "Analysis of direct-detection DPSK signal with homodyne crosstalk," J. Lightw. Technol., vol. 23, no. 9, pp. 2681-2686, Sep. 2005.

[12] T. Kamalakis and T. Sphicopoulos, "Performance analysis of differential-phase-shift-keying optical receivers in the presence of in-band crosstalk noise," J. Lightw. Technol., vol. 23, no. 12, pp. 4084-4092, Dec. 2005.

[13] C. X. Yu, W. Wang, and S. D. Brorson, "System degradation due to multipath coherent crosstalk in WDM network nodes," J. Lightw. Technol., vol. 16, no. 8, pp. 1380-1386, Aug. 1998.

[14] S. D. Dods, J. P. R. Lacey, and R. S. Tucker, "Performance of WDM ring and bus networks in the presence of homodyne crosstalk," $J$. Lightw. Technol., vol. 17, no. 3, pp. 388-396, Mar. 1999.

[15] J. J. O. Pires and L. G. C. Cancela, "A contribution to study the effect of multipath coherent crosstalk due to optical network nodes on DPSK signals," IEEE Photon. Technol. Lett., vol. 21, no. 20, pp. 1499-1501, Oct. 2009.

[16] J. J. O. Pires and L. G. C. Cancela, "Simplifying the error probability analysis in optical direct detection DPSK systems," IEEE Commun. Letters, vol. 13, no. 6, pp. 369-371, Jun. 2009.

[17] G. Contestabile, R. Proietti, N. Calabretta, M. Presi, A. D’Errico, and E. Ciaramella, "Simultaneous demodulation and of 40-Gb/s NRZ-DPSK signals using a multiwavelength Gaussian filter," IEEE Photon. Technol. Lett., vol. 20, no. 10, May 2008.
[18] R. Khosravani, M. I. Hayee, B. Hoanca, and A. E. Willner, "Reduction of coherent crosstalk in WDM add/drop multiplexing nodes by bit pattern misalignment," IEEE Photon. Techno. Lett., vol. 11, no. 1, pp. 134-136, Jan. 1999.

[19] Y. Ma and T. J. Lim, "Bit error probability for MDPSK and NCFSK over arbitrary Rician fading channels," IEEE J. Sel. Areas Commun., vol. 18, no. 11, pp. 2179-2189, Nov. 2000.

[20] J. J. O. Pires and L. G. C. Cancela, "Estimating the performance of direct-detection DPSK in optical networking environments using eigenfunction expansion techniques," J. Lightw. Technol., vol. 28, no. 13, pp. 1994-2003, Jul. 2010.

[21] G. Bosco and P. Poggiolini, "The impact of receiver imperfections on the performance of optical direct-detection DPSK," J. Lightw. Technol., vol. 23, no. 2, pp. 842-848, Feb. 2005.

[22] J. G. Proakis, Digital Communications, 3rd ed. Tokyo, Japan: McGraw-Hill, 1995.

[23] T. T. Tjhung, C. Loo, and N. P. Secord, "BER performance of DQPSK in slow Rician fading," Electron. Lett., vol. 28, no. 18, pp. 1763-1765, Aug. 1992.

[24] P. A. Humblet and M. Azizoĝlu, "On the bit error rate of lightwave systems with optical amplifiers," J. Lightw. Technol., vol. 9, no. 11, pp. 1576-1582, Nov. 1991.

[25] R. E. Ziemer and T. B. Welch, "Equal-gain combining of multichannel DPSK in Doppler-spread Ricean fading," IEEE Trans. Veh. Technol., vol. 49, no. 5, pp. 1846-1855, Sep. 2000.

[26] I. S. Gradshteyn and I. M. Ryzhik, Table of Integrals, Series, and Products. Orlando, FL: Academic, 1980.

João J. O. Pires received the Ph.D. degree in electrical and computer engineering from the Technical University of Lisbon, Portugal, in 1993.

$\mathrm{He}$ is currently an Assistant Professor at Instituto Superior Técnico, Technical University of Lisbon. From 1991 to 1999 he worked on a number of European-funded projects, including RACE and ACTS projects. He is the author or coauthor of more than 60 papers in international journals and conference proceedings. At present his research interests are mainly in the area of optical transport networks and optical broadband access networks.

Luís G. C. Cancela received the Licenciatura and the M.Sc. degrees both in electrical and computer engineering from the Technical University of Lisbon, Portugal, in 1993 and 1998, respectively. He also received the Ph.D. degree in telecommunications from Instituto Superior das Ciências do Trabalho e da Empresa (ISCTE), Lisbon, Portugal in 2008.

Currently, he is a Researcher at Instituto de Telecomunicações in Lisbon and an Assistant Professor at ISCTE. His research interests include the study of optical crosstalk in fiber-optic communication. 